\title{
GENERALIZED HERMITE POLYNOMIALS FOR IMAGE RECONSTRUCTION FROM ZERO CROSSING CONTOURS
}

\author{
Y.V.Venkatesh \\ Computer Vision and Artificial Intelligence Laboratory \\ Department of Electrical Engineering \\ Indian Institute of Science \\ Bangalore-560012, India. \\ email: yvvele@iris.ee.iisc.ernet.in or yvvele@ee.iisc.ernet.in
}

\begin{abstract}
Generalized Hermite polynomials in two variables are employed for the reconstruction of images from a knowledge of their zero crossing contours. The problem of reconstruction of signals as functions of two variables is not a mere extension of that of a single variable. This is a consequence of the fact that the spatial and spectral characteristics of two-variable functions are quite distinct from what one can expect from their separate projections on to the coordinate axes. One of the results of the paper is that we cannot guarantee uniqueness in reconstruction unless we impose certain constraints on, for instance, space-bandwidth products/ratios in the $x-\omega_{x}, y-\omega_{y}$ directions, of the unknown image. Further, a distinguishing feature of the proposed approach is that the standard assumption of bandlimitedness is not invoked. The proposed framework is believed to provide a more unified procedure for signal reconstruction (of uni- and multi-dimensional signals) from partial information than most of the results of the literature. For lack of space, only the main analytical and computational results are presented.
\end{abstract}

Indexing Terms: Hermite polynomials, Image representation, Image reconstruction, Scale Space Analysis, Zerocrossings

\section{INTRODUCTION}

In image processing, there are situations, arising in optics (astronomy, in particular), crystallography, vision and other fields, in which only partial information is available, and the complete signal has to be reconstructed for analysis of its information content. Such problems belong to the class of inverse problems, among which the recovery of an image from the

- magnitude of Fourier data (also known as the phase retrieval problem) ;

- signed transform magnitude ;

- signum of the phase ; and

- zero crossing contours,

have been dominant in the literature. Zero crossing contours are obtained by operating on the image using, for instance, the Laplacian-of-a-Gaussian (LoG) - mainly motivated by a model of the human visual system filtered image, parametrized by $t>0$, with standard deviation, $\sqrt{2 t}[1]$. More precisely, image data $f(x, y)$ is filtered with LoG to get the scale-space functions, $h(x, y, t)$, whose zero set of $h(x, y, t)$ is the point set in $(x, y, t)$ where $h=0$. At a single level, we obtain the zero crossing contours, which are the subject of study in the present paper.

Marr $[1,2]$ and others investigated the relevance of these contours obtained from independent spatial-frequency channels to the early stages of visual information processing. It was suggested that they could form the foundation for a 'primal sketch'. In this context, an important problem is the determination of conditions under which one can reconstruct an image from these zero crossing contours. This problem has led to considerable investigations by researchers in signal processing. If there exists a solution to this problem, we also need to know an algorithm to achieve reconstruction from the given partial information.

\subsection{Literature in Brief}

The literature on the above problem is quite extensive. In view of space limitations, we include here only relevant references. For more details and a critical review, see [14].

In the course of solving the problem, it turns out that we encounter the basic issue of image representation which is to be: complete, stable, and explicit in feature description (like gradients, edges, and corners).

When the image data, $f(x, y)$ (and hence the Laplacian of the image data, $\Delta f(x, y, t))$ are polynomial, the authors of $[4,5,8,9]$ invoke results from algebraic geometry for solving the problem of reconstruction. In the last quoted reference, a zero crossing contour is expanded, at two points of the zero crossing in scale space, using its arbitrarily high order derivatives. In the same algebraic geometry framework, the authors of $[4,6]$ extend the above results to the case when $f(x, y)$ is a band-limited function (having a finite Fourier series) which is irreducible as an entire function. It is appropriate to remark here that (i) the bandpass characteristics of two-dimensional signals cannot be analysed in terms of their projections on frequency axes; and (ii) in the literature, uniqueness conditions for reconstruction from mag- 
nitude and from sign data involve the factorization of entire functions of exponential type.

In [7], the authors consider a band-limited image, $f(x, y)$, and employ the precise locations in the $(x, y)$ domain where $f$ crosses some threshold (such as zero, assuming that $f$ has both positive and negative regions).

An image can be treated as a stacking of one-dimensional scan lines, and the mathematical preliminaries presented for one-dimensional signal analysis can, in principle, be extended to image analysis. This is, in fact, the approach of [?], in which the authors extend the Logan theorem [10] for application to images. To this end, they decompose the image into a sum of images, each componenet of which is confined to a bandpass region with a width of one octave or less in at least one of the two spectral dimensions. However, the low frequency components are omitted from the reconstruction process, and simply stored as a part of the representation process.

According to some authors, a natural framework for the analysis of scale-space formulations of multi-resolution representations is in terms of the heat equation. Hummel and Moniot [8] reconstruct the function $h$ in scale space, and make use of the fact that $h$ satisfies the heat equation. According to them, zero crossings, when supplemented with gradient data along the zero crossing boundaries, are sufficient (in theory) to reconstruct the function $\Delta f(x, y)$ (and, under suitable functions, the original function $f(x, y)$, by solving the Poisson equation).

\section{MATHEMATICAL PRELIMINARIES}

A motivation for the present work comes from the fact that, in practice, signals, which are spatially finite are not strictly finite in extent in the spectral domain (see [18]). In view of the finite field of view of biological vision systems, and in order to develop a consistent mathematical theory, images can be treated as though they are approximately infinite in both the spatial and time domains.

Further, physical systems are not 'bandlimited' in the strict sense required by the familiar mathematical condition on the spectrum (or Fourier transform) of its impulse response. Signals generated or output by them cannot also be strictly bandlimited (see [18]). Observe also that the Laplacian-of-a-Gaussian (LOG) mask does not, strictly speaking, correspond to a perfect bandpass filter.

In order to represent an image, we employ generalized Hermite polynomials which constitute a complete orthogonal basis for a class of $L_{2}$-functions. Using this representation, we reconstruct an image from the given zero crossing contours, under certain conditions on the spatial/spectral spread of the unknown image. Since images are, in practice, spatially finite, they are not strictly finite in extent in the spectral domain. In order to develop a consistent mathematical theory, we treat images as having unbounded support in both the spatial and time domains, but, more importantly, we use some measures to indicate the extent of effective signal spread in these domains.

Images, which are treated as two-dimensional functions, are assumed to be defined over $(-\infty, \infty) \times(-\infty, \infty)$ in both the spatial and spectral domains. Let $f(x, y) \in L^{2}(\mathcal{R})$ be a real-valued function of $x, y \in \mathcal{R}$ with the Fourier transform,
$F\left(j \omega_{1}, j \omega_{2}\right)$. The two functions, $f(x, y)$ and $F\left(j \omega_{1}, j \omega_{2}\right)$ form a Fourier integral pair. The classical uncertainty principle says that they cannot both have compact support or, in other words, cannot be highly concentrated in a finite region of the $(x, y)$ and the $\left(\omega_{1}, \omega_{2}\right)$-domains [22]. A measure of localization is effective width. We define the effective spread in the $x$-direction, around $\left(x_{0}, y_{0}\right)$, and that in the $\left(\omega_{1}\right)$-direction, around $\left(\omega_{10}, \omega_{20}\right)$, by

$$
X_{e}=\sqrt{\frac{1}{E} \int_{-\infty}^{\infty} \int_{-\infty}^{\infty}\left(x-x_{0}\right)^{2}|f(x, y)|^{2} d x d y}
$$

$\Omega_{1 e}=\sqrt{\frac{1}{4 \pi^{2} E} \int_{-\infty}^{\infty} \int_{-\infty}^{\infty}\left(\omega_{1}-\omega_{10}\right)^{2}\left|F\left(\omega_{1}, \omega_{2}\right)\right|^{2} d \omega_{1} d \omega_{2},}$

where $E$ denotes the integral of $|f|^{2}$ We can obtain similar expressions for the effective spreads in the other directions Using the Schwarz inequality, we can arrive at the the uncertainty inequalities in two dimensions. It is interesting to note that these come only pairwise, along the corresponding axes. Thus we have the space-bandwidth-products satisfying the inequalities: $X_{e} \Omega_{1 e} \geq \frac{1}{4}$ and $Y_{e} \Omega_{2 \varepsilon} \geq \frac{1}{4}$, while $X_{e} \Omega_{2 e}$ can vanish. Functions which have finite effective widths in the spectral domains are said be essentially bandlimited (or bandpass). For purposes of symmetry, the functions can be said to be essentially space-limited in the spatial domains, too. (In what follows, the subscript, e, denoting "effective" will be omitted.)

\subsection{Generalized Hermite Polynomials}

Consider the generalized one-dimensional Hermite polynomials, parametrized by $\sigma$, and generated as follows :

$H_{n}(z, \sigma)=\left.(-1)^{n} e^{\frac{x^{2}}{2}} \frac{d^{n}}{d x^{n}}\left(e^{-x^{2}}\right)\right|_{x=\frac{z}{\sqrt{\sigma}}}$, for $n=0,1,2,3, \ldots$

It is known [20] that the $H_{n}$ 's form a complete basis for the class $C$ of real functions, $\psi(x)$, defined on the infinite interval $(-\infty, \infty)$, which are piecewise continuous in every finite sub- interval $[-a, a]$, and satisfy the condition,

$$
\int_{-\infty}^{\infty}\left(1+x^{2}\right) e^{\frac{-x^{2}}{\sigma}} \psi^{2}(x) d x<\infty
$$

The generalized one-dimensional Hermite polynomials parametrized by $\sigma$, can be used to generate (by tensor product) the two-dimensional versions, parametrized by $\sigma_{1}$ and $\sigma_{2}$ :

$$
H_{m, n}\left(x, y, \sigma_{1}, \sigma_{2}\right)=P_{m}\left(x, \sigma_{1}\right) P_{n}\left(y, \sigma_{2}\right) e^{-\left(\frac{x^{2}}{2 \sigma_{1}}\right)-\left(\frac{y^{2}}{2 \sigma_{2}}\right)}
$$

for $m, n=0,1,2, \ldots, \infty$, where $P_{m}\left(x, \sigma_{1}\right)$ and $P_{n}\left(y, \sigma_{2}\right)$ are polynomials in $x, y$. 


\subsection{Image Representation}

We use the vector notation, $\underline{p}$, to denote (at times, for convenience, when no explicit reference to a particular spatial variable is required) the variables $\left(x, y, \sigma_{1}, \sigma_{2}\right)$ as a whole. For typical polynomials, their recurrence relations, and Fourier transforms, see [14].

The parameters $\sigma_{1}, \sigma_{2}$ control the effective width of the signal in both the spatial and frequency domains - the smaller the values of $\sigma_{1}$ and $\sigma_{2}$, smaller the spatial width (and greater the spectral width), and vice versa, in the the directions of $\mathrm{x}$ and $\mathrm{y}\left(\omega_{1}\right.$ and $\left.\omega_{2}\right)$ respectively.

In what follows, $\sum_{m, n}$ denotes summation with respect to $\mathrm{m}$ and $\mathrm{n}$, each ranging, unless otherwise indicated, from 0 to $\infty$. Let the $L^{2}$-norm squares of these polynomials be denoted by $k_{m, n}$, for $m, n=0,1,2, \ldots, \infty$

Now, we define formally the series,

$$
f(x, y)=\sum_{m, n} \gamma_{m, n} H_{m, n}(\underline{p}), \quad-\infty<x, y<\infty
$$

where the coefficients $\gamma_{m, n}$ are calculated from the relation,

$\gamma_{m, n}=\frac{1}{k_{m, n}} \int_{-\infty}^{\infty} \int_{-\infty}^{\infty} f(x, y) H_{m, n}(\underline{p}) d x d y, \quad m, n=0,1, \ldots, \infty$

For theoretical details on convergence, and derivation of effective spreads in the spatial and spectral domains, see [14]. It can be shown that the effective spatial width in the $\mathrm{x}$-direction is given by

$$
X_{s p a t i a l}^{2}=\frac{1}{4} \sigma_{1} \frac{\sum_{m, n}\left[2(m+1) \gamma_{m+1, n}+\gamma_{m-1, n}\right]^{2} * A_{m, n}}{\sum_{m, n} \gamma_{m, n}^{2} * A}
$$

where $A_{m, n}$ is a constant equal to the integral of $\left|H_{m, n}\right|^{2}$. Similar results can be obtained for the effective spectral width, $\Omega_{1}$, and for the effective cross-spatial, and crossspectral widths. See [14] for the derivation of expressions related to the effective space-bandwidth product and spacebandwidth ratio. It is found, for instance, that the spacebandwidth ration in the $x$-direction is directly proportional to $\sigma_{1}$, the constant of proportionality being governed by the ratio of the two quadratic forms, involving the coefficients, $\gamma_{m, n}$ of the expansion. It is important to that the complex version of the representation scheme is an entire function of order 2, and type, $\frac{1}{\left(2 \sigma_{1}\right)}$ in the $z_{1}$ domain, and $\frac{1}{\left(2 \sigma_{2}\right)}$ in the $z_{2}$ domain.

\subsection{Zero Crossing Contours}

The Laplacian of the image function represented by the generalized Hermite polynomials at a fixed scale $\left(\sigma_{1}, \sigma_{2}\right)$ can be shown to reduce to:

$$
\begin{gathered}
\left(\frac{\partial^{2}}{\partial x^{2}}+\frac{\partial^{2}}{\partial y^{2}}\right) f(x, y)=\sum_{m, n} \gamma_{m, n}\left(\frac{\partial^{2}}{\partial x^{2}}+\frac{\partial^{2}}{\partial y^{2}}\right) H_{m, n} \\
=\sum_{m, n} \mu_{m, n} H_{m, n}
\end{gathered}
$$

where $\mu_{m, n}=$

$$
\begin{array}{r}
\sum_{m, n} \frac{1}{4 \sigma_{1}} \gamma_{m-2, n}+\frac{1}{4 \sigma_{2}} \gamma_{m, n-2}-\left(\frac{1}{2 \sigma_{1}}+\frac{1}{2 \sigma_{2}}+\frac{m}{\sigma_{1}}+\frac{n}{\sigma_{2}}\right) \gamma_{m, n} \\
+\frac{(m+2)(m+1)}{\sigma_{1}} \gamma_{m+2, n}+\frac{(n+2)(n+1)}{\sigma_{2}} \gamma_{m, n+2}
\end{array}
$$

From (10), we can directly extract, analyse (and synthesize) the zero crossing contours at the desired scale of representation.

\section{MAIN RESULTS}

We consider the following specific cases of image reconstruction from zero crossing contours:

1. Suppose the zero crossing contours of the unknown image function, $f(x, y)$, are given by:

$$
\phi(x, y)=\sum_{m=0, n=0}^{m=M, n=N} \lambda_{m, n} x^{m} y^{n}=0 .
$$

Then the attempted representation of $f(x, y)$ is:

$f(x, y)=\sum_{m=0, n=0}^{m=M, n=N} \alpha_{m, n} x^{m} y^{n} P_{m}\left(x, \sigma_{1}\right) P_{n}\left(y, \sigma_{2}\right) e^{-\left(\frac{x^{2}}{2 \sigma_{1}}\right)-\left(\frac{y^{2}}{2 \sigma_{2}}\right)}$

for $m=0,1,2, \ldots, M$, and $n=0,1,2, \ldots, N$, where (see (3)) $P_{m}\left(x, \sigma_{1}\right)$ and $P_{n}\left(y, \sigma_{2}\right)$ are polynomials in $x, y$. The zero crossing contours of this function are given by:

$$
\sum_{m=0, n=0}^{m=M, n=N} \alpha_{m, n} x^{m} y^{n} P_{m}\left(x, \sigma_{1}\right) P_{n}\left(y, \sigma_{2}\right)=0,
$$

which, on comparison and matching of corresponding terms with (8), will lead to the following set of equations in the unknown coeficients $\alpha_{m, n}$, and the variance (or scale) parameters, $\sigma_{1}, \sigma_{2}$ :

$$
\mathcal{A} \underline{\alpha}=\underline{\lambda}
$$

where $\mathcal{A}$ is a $(M+1)(N+1) \times(M+1)(N+1)$ matrix of known elements, $\underline{\alpha}$ is the $(M+1)(N+1)$ - vector of lexicographically ordered set of unknown coefficients, $\alpha_{m, n}$, and $\underline{\lambda}$, is the correspondingly (lexicographically) ordered set of known coefficients, $\lambda_{m, n}$. Since $\sigma_{1}$ and $\sigma_{2}$ are unknown, and hence can be chosen arbitrarily, solution of (9) for $\underline{\alpha}$ is non-unique. Therefore, the reconstruction is nonunique. In other words, any number of image functions exist with exactly the same zero crossing contours. See Fig. 1 for a typical synthetic image which has the same zero crossing contour. The ambiguity in the values of $\sigma_{1}$ and $\sigma_{2}$ can be overcome by one of the following additional input data sets:

(a) Space-Bandwidth Products in the $\left(x-\omega_{1}\right)$, and $\left(y-\omega_{2}\right)$ pairs, which would give two additional equations. 
(b) Space-Bandwidth Ratios in the $\left(x-\omega_{1}\right)$, and $\left(y-\omega_{2}\right)$ pairs, which would give two additional equations, explicitly involving $\sigma_{1}$ and $\sigma_{2}$.

(c) A combination of the above two cases (SpaceBandwidth Product and Space-Bandwidth Ratio) to create two additional equations.

(d) $x$ - and $y$-gradient information at any one point say, $\left(x_{1}, y_{1}\right)$, of the zero-crossing contour. Then, from the above representation of $f(x, y)$, we get:

$$
\begin{gathered}
\left.\frac{\partial f(x, y)}{\partial x}\right|_{x=x_{1}, y=y_{1}}= \\
=\left.\left(\frac{\partial \phi}{\partial x}-\phi(x, y) \frac{x}{\sigma_{1}}\right) e^{-\left(\frac{x^{2}}{\sigma_{1}}\right)-\left(\frac{y^{2}}{\sigma_{2}}\right)}\right|_{x=x_{1}, y=y_{1}} \\
=\left.\left(\frac{\partial \phi}{\partial x}\right) e^{-\left(\frac{x^{2}}{\sigma_{1}}\right)-\left(\frac{y^{2}}{\sigma_{2}}\right)}\right|_{x=x_{1}, y=y_{1}} .
\end{gathered}
$$

A similar equation is derived from a knowledge of the $y$-gradient at a point on the zero-crossing contour. The two resulting equations can be solved for the the unknowns, $\sigma_{1}$ and $\sigma_{2}$.

See Fig. 2 for a typical result of reconstruction of an image from (synthetic) zero crossing contours, with prescribed values of $\sigma_{1}$ and $\sigma_{2}$.

2. If the zero crossing contours of $\psi(x, y)=\nabla^{2} f(x, y)$ are given by (8), then the results of Case 1 above are valid for $\psi(x, y)$. However, if $f(x, y)$ is to be obtained directly, then there two ways of handling the situation:

(a) Solve Poisson's equation for $f(x, y)$ from the solution for $\psi(x, y)$ of Case 1

(b) A more direct solution involves the utilization of (15), from which we obtain the set of equations of the form (17), with the matrix $\mathcal{A}$ now of size, $(M+3)(N+3) \times(M+1)(N+1)$, and the unknown coefficient vector $\underline{\alpha}$, containing $(M+$ $3)(N+3)$ elements (apart from the unknown $\sigma_{1}$, and $\sigma_{2}$ ).

Additional equations are to be created from a knowledge of (i) Space-Bandwidth Products and Space-Bandwiclth Ratios; and (ii) $x$ - and $y-$ grad values at $2 M+2 N-2$ (minimal) points on the zero crossing contours.

3. If the zero crossing contours of

$$
\Psi(x, y)=\nabla^{2} f(x, y) * g(x, y, \mu)
$$

are given by (8), then the results of Case 1 above are valid for $\Psi(x, y)$. If $f(x, y)$ is to be obtained directly, then knowledge of $\mu$ is to be presumed. However, this case is quite complicated. A possible solution is to treat the problem as one of deblurring (in addition to reconstruction from zero crossing contours). See [23] for a new approach to deblurring.

\section{CONCLUSIONS}

A new framework, involving the generalized Hermite polynomials, has been proposed for the reconstruction of images from a knowledge of its zero crossing contours. Since the spatial and spectral characteristics of an image functions are quite distinct from what one can expect from their separate projections on to the coordinate axes, the problem of reconstruction is a nontrivial extension of the problem corresponding to one-variable functions. The framework proposed in the paper dispenses with the standard assumption of bandlimitedness, and seems to provide a more unified procedure for signal reconstruction of both uni- and multi-dimensional signals from partial information than most of the results of the literature. It is shown that we cannot guarantee uniqueness in image reconstruction unless we impose certain constraints on, for instance, space-bandwidth products/ratios in the $x-\omega_{x}, y-\omega_{y}$ directions, of the unknown image. For lack of space, only the main analytical (along with some computational) results are presented. The final paper is to (i) contain a more complete derivation of analytical results; and (ii) feature the use of simulated annealing for optimal reconstruction. Application of the proposed framework to reconstruction of images from other types of partial information, as also to scale-space filtering will appear elsewhere.

\section{REFERENCES}

[1] D.Marr, Vision, San Francisco, CA : Freeman, 1982.

[2] D.Marr, S.Ullman and T.Poggio, "Bandpass Channels, Zero-crossings, and Early Visual Information Processing," J. Opt. Soc. Am., Vol.69, No.6, pp 914-916, June 1979.

[3] A.Witkin, "Scale space filtering," Proc. Int. Joint Conf. Artificial Intell. 1983.

[4] J. L. C. Sanz and T. Huang, "Image representation by sign information," IEEE Trans. Pattern Analysis and Machine Intelligence, Vol. 11, pp. 729 - 738, 1989.

[5] S.R.Curtis, A.V.Oppenheim and J.S.Lim, "Signal Reconstruction from Fourier Transform Sign Information," IEEE Trans. ASSP, Vol.33, No.3, pp 643-657, June 1985.

[6] S. Curtis, S. Shitz, and A. Oppenheim, "Reconstruction of periodic two-dimensional signals from zero crossings," IEEE Trans. Acoust. Speech, and Signal Processing, Vol. ASSP-35, pp. 890-897, 1987.

[7] S. Curtis and A. Oppenheim, "Reconstruction of multidimensional signals from zero crossings,", J. Opt. Soc. Amer., Vol. 4, pp. 221 - 230, 1987.

[8] R. Hummel and R. Moniot, "Reconstruction from zero crossings in scale space,", IEEE Trans. Acoustics, Speech, and Signal Processing, Vol. 37, pp. 2111 - 2130, 1989.

[9] A. Yuille and T. Poggio, "Fingerprint theorems for zero crossings," J.Opt. Soc. Amer., Vol.2, No.5, pp 683-692, May 1995 
[10] "Information in the zero crossings of bandpass signals,", Bell System Technical Journal, Vol. 56, p. 510, 1977.

[11] Y. Y. Zeevi and D. Rotem, "Image reconstruction from zero crossings," IEEE Trans. Acoustics, Speech and Signal Processing, Vol. ASSP-34, pp. 1269 - 1277, 1986.

[12] Y.O.Al-Jalili, "Analysis and detection algorithms for complex time zeros of bandlimited signals," IEE Proceedings-I, Vol.138, No.3, pp 189-200, June 1991.

[13] G.B.Lockhart and Y.O.Al-Jalili, "Method for superimposing data on amplitude modulated signals," Electronics Letters, Vol.18, No.9, pp 379-381, 1982.

[14] Y. V. Venkatesh, "Reconstruction of images from zero crossing contours," Technical Report, Department of Electrical Engineering, Indian Institute of Science, Bangalore, November 1997.

[15] Y. V. Venkatesh, "Hermite polynomilas for signal reconstruction from zero crossings, Part I: Onedimensional signals,", IEE Proc.-I (Communications, Speech and Vision), Vol. 139, No. 6, pp. 587-596, 1992.

[16] R. P. Boas, Entire Functions, Academic Press, p. 65, and p. 113, 1954.
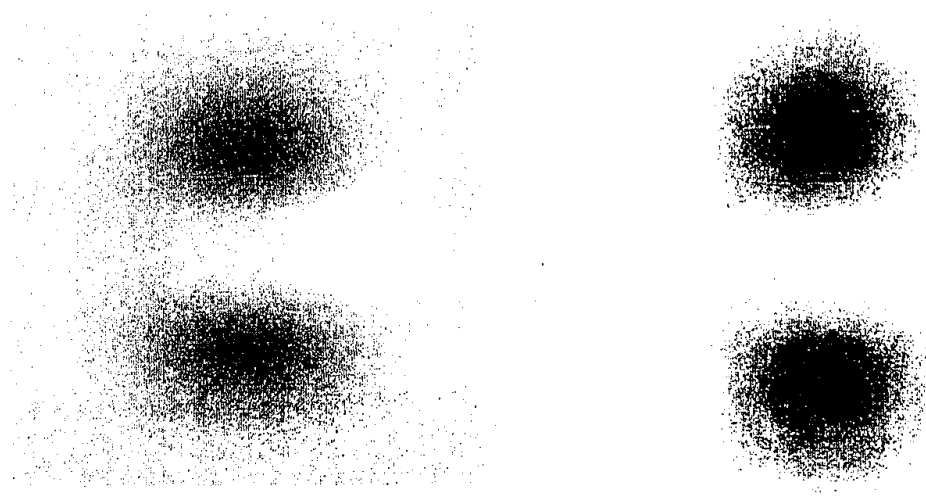

Fig.1(a) \& (b) Synthetic images with the same zero

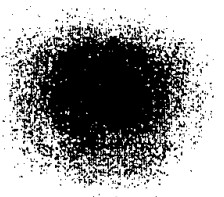
crossing contours

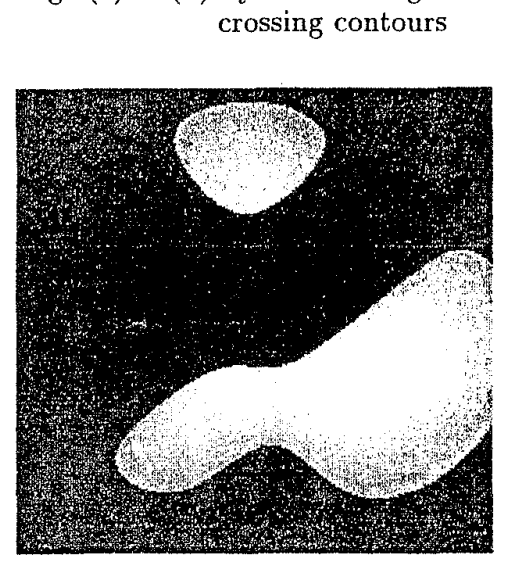

Fig.2(a) Original Synthetic Image
[17] J. L. Sanz, "Multidimensional signal representation by zero crossings: an algebraic study,", SIAM J. Appl. Math., Vol. 49, No. 1, pp. 281-295, 1989.

[18] D. Slepian, "On bandwidth," Proc. IEEE, Vol. 64, pp. 292-300, 1976.

[19] J. G. Daugman, "Uncertainty relation for resolution in space, spatial frequency, and orientation optimized by two-dimensional visual cortical filters," J. Opt. Soc. America A, Vol. 2, No. 7, pp. 1160 - 1169, 985.

[20] N.N.Lebedev, Special Functions and Their Applications, Dover Publications, Inc. New York , pp 60-76, 1972.

[21] J.R.Higgins, "Completeness and Basis Properties of Sets of Special Functions," Cambridge, Cambridge University Press, Great Britain, 1977.

[22] D.L.Donoho and P.B.Stark, "Uncertainty principles and signal recovery," SIAM J1. Appl. Math., Vol.49, No.3, pp 906- 931, June 1989.

[23] M. Vairy and Y. V. Venkatesh, "Deblurring Gaussian blur using a wavelet array transform,", Pattern Recognition, Vol. 28, No. 7, pp. 965-976, 1995.

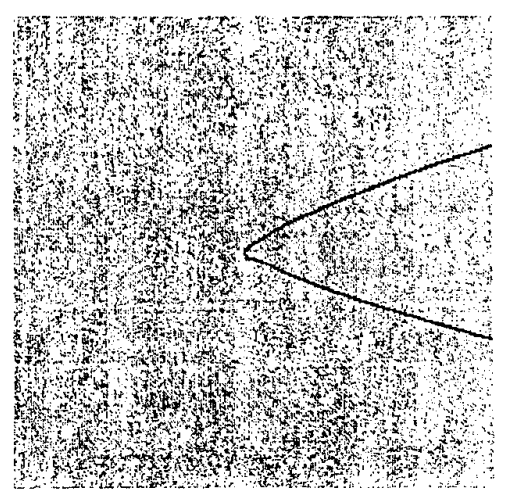

Fig. 1(c) Common Zero Crossing Contour

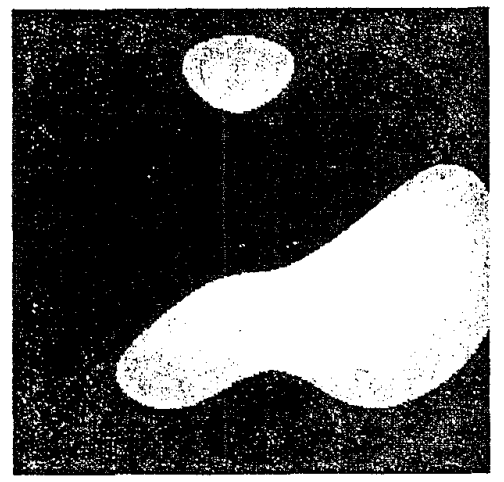

Fig. 2(b) Its reconstruction from zero crossing contours 